BULL. AUSTRAL. MATH. SOC.

VOL. 31 (1985), 159-160.

\title{
AN IMPROVED CONVEXITY MAXIMUM PRINCIPLE \\ AND SOME APPLICATIONS
}

\section{ALAN U. KENNINGTON}

This thesis investigates geometric properties of a class of elliptic boundary value problems. Geometric assumptions are made on the data defining the problems in order to prove geometric properties of the solutions.

The problems considered are of the type

$$
\begin{aligned}
a_{i j}(\nabla u) u_{i j}+b(x, u, \nabla u) & =0 \text { in } \Omega, \\
u & =0 \text { on } \partial \Omega,
\end{aligned}
$$

where $\Omega$ is a bounded convex domain in $\mathbb{R}^{n}$ for some $n \geq 2,\left[u_{i j}\right]$ is the matrix of second derivatives of a function $u$ on $\Omega,\left[a_{i j}\right]$ is a positive semi-definite real symmetric matrix, and $b>0$. (The usual summation convention is understood.) The geometric properties considered are generalisations of concavity.

The principal results are a convexity maximum principle and a concavity theorem. The maximum principle is an improved version of one proved by Korevaar ([1]). It states that if a function $v$ on $\Omega$ is smooth enough and satisfies (1) with $b(x, z, p)^{-1}$ a convex function of the joint variable $(x, z)$ for all $p$, then the function $c(x, y)$

Received 26 October 1984. Thesis submitted to University of Adelaide, February 1984. Degree approved October 1984. Supervisor: Dr J.H. Mi chael.

Copyright Clearance Centre, Inc. Serial-fee code: 0004-9727/85 $\$ A 2.00+0.00$. 
defined on $\Omega \times \Omega$ by

$$
c(x, y)=(1-\lambda) v(x)+\lambda v(y)-v((1-\lambda) x+\lambda y)
$$

cannot take on a positive maximum in the interior of $\Omega \times \Omega$. Appropriate boundary behaviour of $v$ guarantees that $c$ is non-positive near the boundary of $\Omega \times \Omega$, from which it follows that $v$ is concave in $\Omega$. The concavity theorem generalises this by substituting $u^{\alpha}$ for $v$ with $0<\alpha \leq 1$. Then $u^{\alpha}$ is shown to be concave in $\bar{\Omega}$ under some rather complicated conditions on $b$.

A special case is Poisson's equation $\Delta u+f(x)=0$. If $f(x)^{\beta}$ is concave in $\Omega$ for some $\beta \geq 1$, then $u(x)^{\alpha}$ is concave with $\alpha=\beta /(1+2 \beta)$. This value of $\alpha$ is shown by counterexamples to be sharp. (Note that if $u^{\alpha}$ is concave then $u^{\gamma}$ is concave whenever $0<\gamma \leq \alpha$. ) Thus the solution is seen to inherit geometric properties from the data.

Since the thesis was submitted, the author has found a simplification of the conditions required of $b(x, z, p)$. The simpler conditions are that $z^{\alpha-1} b(x, z, p)$ be decreasing with respect to $z$, and $z^{(3 \alpha-1) / \alpha} b\left(x, z^{1 / \alpha}, z^{(1-\alpha) / \alpha} p\right)$ be jointly concave with respect to the variable $(x, z)$ in $\Omega \times(0, \infty)$.

At the time of writing the thesis, only a small handful of articles had appeared on this topic. Now there is an extensive, rapidly growing literature on the concavity-like properties of solutions of boundary value problems.

\section{Reference}

[1] N.J. Korevaar, "Convex solutions to nonlinear elliptic and parabolic boundary value problems", Indictiniv. Math. J. 32 (1983), 603-614.

Department of Mathematics,

University of Melbourne,

Parkville, Victoria 3052, Australia. 